\title{
Short-term variations in photosynthetic parameters of Nannochloropsis cultures grown in two types of outdoor mass cultivation systems
}

\author{
Jacco C. Kromkamp ${ }^{1, *}$, John Beardall ${ }^{2}$, Assaf Sukenik ${ }^{3}$, Jiři Kopecký ${ }^{4}$, \\ Jiři Masojidek ${ }^{5}$, Stef van Bergeijk ${ }^{6}$, Shai Gabai ${ }^{7}$, Efrat Shaham ${ }^{7}$, Amir Yamshon ${ }^{7}$ \\ ${ }^{1}$ Netherlands Institute of Ecology, Centre for Estuarine and Marine Ecology (NIOO-CEME), PO Box 140, 4400 AC Yerseke, \\ The Netherlands \\ ${ }^{2}$ School of Biological Sciences, Monash University, Wellington Road, Clayton, Victoria 3800, Australia \\ ${ }^{3}$ Israel Oceanographic and Limnological Research, Yigal Allon Kinneret Limnological Laboratory, PO Box 447, \\ 14950 Migdal, Israel \\ ${ }^{4}$ Institute of Physical Biology, University of South Bohemia, Zámek 136, 37333 Nové Hrady, Czech Republic \\ ${ }^{5}$ Institute of Microbiology, Academy of Sciences, Opatovický mlýn, 37981 Třeboň, Czech Republic \\ ${ }^{6}$ IFAPA Centro El Toruño Ctra. N IV Madrid-Cádiz, km 654a Camino de Tiro Pichón, 11500 El Puerto de Santa María, Spain \\ ${ }^{7}$ The Mina and Everard Goodman Faculty of Life Sciences, Bar-Ilan University, Ramat-Gan 52900, Israel
}

\begin{abstract}
The present study evaluated productivity under different operational (optical) conditions using the unicellular alga Nannochloropsis sp. (Eustigmatophyta). This alga is often grown in mass algal cultures because it contains high contents of the polyunsaturated fatty acid, eicosapentaenoic acid (EPA, 20:5 $\omega 3$ ). We followed biomass, photosynthetic activities and physiological parameters in a $3 \mathrm{~d}$ experiment using various photosynthesis techniques in 2 outdoor cultivation systems: a flat panel photobioreactor (FPP) and a high rate algal pond (HRAP). We aimed to detect acclimation behaviour to the different optical conditions, with the FPP having a short light path and the HRAP having a long light path. Both algae cultures showed an afternoon depression of photosynthetic activity, which was more pronounced in the FPP. Therefore, the HRAP culture showed 'classical' shade adaptation behaviour, whereas the FPP algae was high-light acclimated. The FPP showed diurnal changes in the potential rates of photosynthesis and respiration. High temperatures $\left(41^{\circ} \mathrm{C}\right)$ on Day 3 in the FPP caused a large reduction in the maximum Photosystem II (PSII) efficiency $\left(F_{\mathrm{v}} / F_{\mathrm{m}}\right)$ and an over-reduction of the PSII acceptors, which did not recover during the light period. Chlorophyll fluorescence measurements with and without dark adaptation suggested that the decreased photosynthetic activity was due to both chronic and dynamic downregulation. Photodamage was higher in the FPP, but most was recovered during the evening. A comparison between the quantum efficiencies for PSII charge separation and oxygen evolution revealed a close coupling between the 2 processes. We also concluded that the optical absorption cross section of PSII equalled $75 \%$ of the total absorption cross section.
\end{abstract}

KEY WORDS: Environmental stress - Flat panel photobioreactor - High rate algal pond . Nannochlopsis · Mass culture · Photosynthetic rate · Chlorophyll fluorescence · Quantum efficiency

\section{INTRODUCTION}

Mass cultures of algae receive considerable attention because of their potential to clean up waste water (Gonzalez et al. 2008, Zhang et al. 2008), yield valu- able products (Nitsan et al. 1999, Zhu \& Jiang 2008), produce high levels of biomass (Gordon \& Polle 2007) and, more recently, be used as a source for biofuels and biodiesel (Gordon \& Polle 2007). Both indoor systems, which use either artificial lighting or sunlight 
(Matthijs et al. 1996), and outdoor systems, which use natural solar radiation, are currently in use. Although operational conditions can be optimized for outdoor production ponds and photobioreactors to maintain maximal production rates for extended periods (Borowitzka 1999), these systems are nonetheless prone to technical failures leading to possible conditions of stress for the algae. For reliable large scale production it is essential that algae can recover from these stressful conditions.

The unicellular alga Nannochloropsis (Eustigmatophyta) was used here with the aim to study a single species and to evaluate productivity under different operational conditions using different methodologies to measure photosynthetic performance. Nannochloropsis is recognized as an excellent source of eicosapentaenoic acid (EPA, 20:5 $\omega 3$ ), a polyunsaturated fatty acid essential for human and animal consumption. Mass production of Nannochloropsis can be easily attained in high rate algal ponds (Sukenik 1999), in flat panel photobioreactors (Zuo \& Richmond 1999) or in tubular photobioreactors (Chini Zittelli et al. 1999).

In the present study we explored the photosynthetic behaviour in 2 optically different outdoor culture systems: a high rate algal pond (HRAP) and a flat panel photobioreactor (FPP). Both systems differ significantly with respect to the optical conditions experienced by the algae. In the HRAP, the high biomass density in the culture creates a steep light gradient where the algae circulate from the surface, with high irradiance, to the bottom of the pond with low irradiance. Although the maximum irradiance experienced might be high, the average irradiance is limited. In an FPP, the light path is often restricted to several centimetres, meaning that the average irradiance is higher than in the HRAP. We were especially interested in the photophysiological acclimation pattern under various optical conditions. The results presented here focused on characterising the normal diurnal fluctuations in physiological performance of the 2 culture types, which acclimate to different irradiance regimes and other physicochemical features experienced in the FPP and HRAP. In an accompanying paper (Sukenik et al. 2009, this Special Issue) we studied the photosynthetic responses of algae using chlorophyll fluorescence methods under high irradiance conditions.

\section{MATERIALS AND METHODS}

Algal mass production systems. Two outdoor mass algal production cultures systems were employed for cultivation of the microalga Nannochloropsis (Fig. 1). The HRAP was a concrete raceway with a surface area of $60 \mathrm{~m}^{2}$, equipped with a paddle wheel (Sukenik et al.
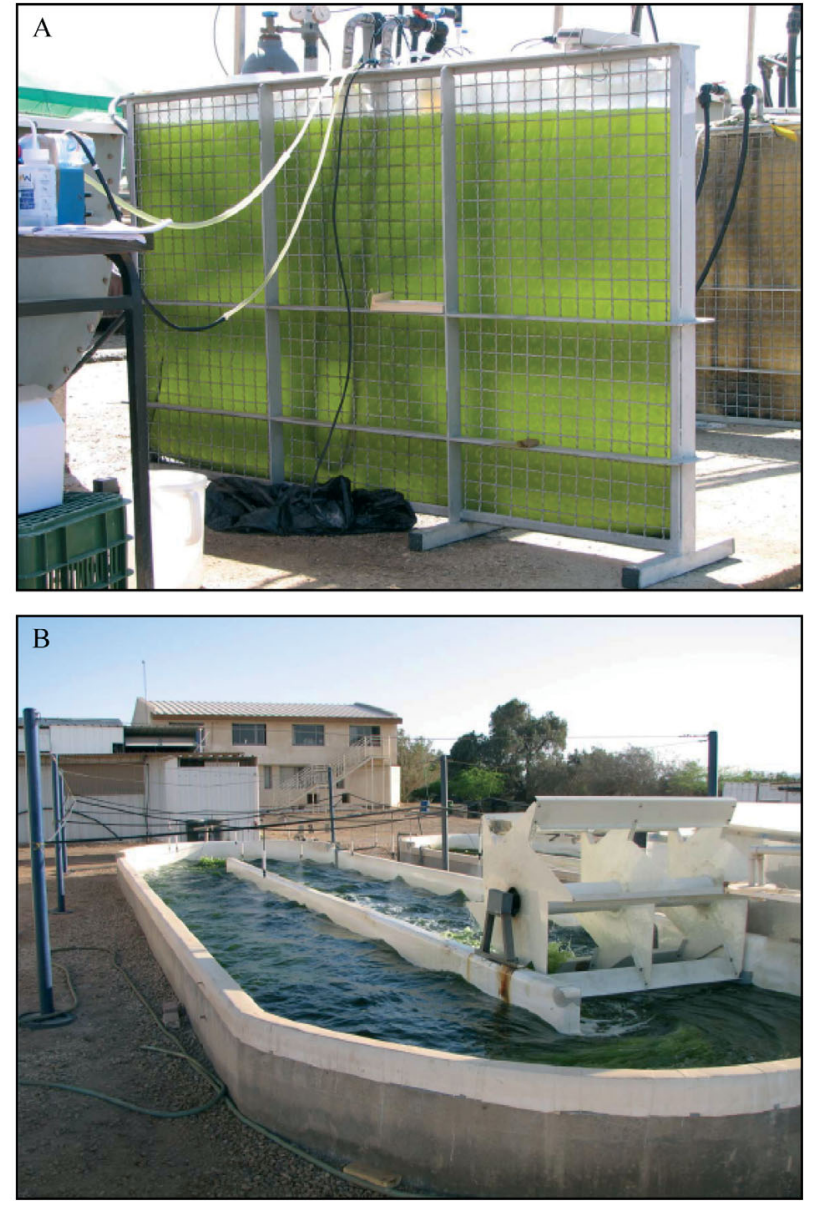

Fig. 1. (A) The flat panel photobioreactor (FPP), constructed of a metal net supporting a flat plastic bag of $5 \mathrm{~cm}$ depth. (B) The high rate algal pond (HRAP), constructed of concrete channels with circulation provided by a paddle wheel

1993), and where the depth of the culture was maintained at $35 \mathrm{~cm}$. To avoid $\mathrm{pH}$ shifts in the dense culture, $\mathrm{CO}_{2}$ was supplied through a solenoid valve activated by a $\mathrm{pH}$ controller set to $\mathrm{pH}$ 7.8. The culture was diluted daily by replacing $10 \%$ of the pond volume with fresh medium. Estimated daily evaporation was compensated for with domestic tap water. The culture medium consisted of $70 \%$ seawater and $30 \%$ tap water to provide a salinity of 28, and the medium was enriched with $2 \mathrm{mM}\left(\mathrm{NH}_{4}\right)_{2} \mathrm{SO}_{4}, 0.2 \mathrm{mM} \mathrm{H}_{3} \mathrm{PO}_{4}$ and $0.02 \mathrm{mM} \mathrm{Fe}_{2}\left(\mathrm{SO}_{4}\right)_{3}$. The HRAP was operated continuously for $4 \mathrm{wk}$ before measurements were taken.

The FPP comprised a metal frame that held a $100 \mathrm{l}$ flat plastic bag, $5 \mathrm{~cm}$ wide, $200 \mathrm{~cm}$ long and $100 \mathrm{~cm}$ high (Fig. 1). The Nannochloropsis culture from the HRAP was used to inoculate the FPP $1 \mathrm{wk}$ before the measurements started. Either air (Days 1 and 2) or $\mathrm{CO}_{2}$-enriched air (ca. $0.5 \% \mathrm{CO}_{2}$, Day 3) was bubbled through the culture at the rate of $51 \mathrm{~min}^{-1}$ to maintain 
high turbulence and keep the $\mathrm{pH}$ in a range between 7.5 and 8.2. The culture $\mathrm{pH}$ and temperature were monitored continuously. Seawater was circulated in 'cooling fingers' immersed in the culture to maintain physiological temperature in the FPP culture.

The study was carried out at the National Mariculture Center (NCM) Israel Oceanographic and Limnological Research, Eilat, Israel, for 3 consecutive days from 1 to 3 April 2008.

Chlorophyll and cell concentrations. Collected cultures were sub-sampled to measure pigment concentration, cell concentration and algal biomass. Photosynthetic pigments were extracted by homogenizing samples in $90 \%$ acetone, then filtered through glass fibre filters and measured using a DW-2a spectrophotometer (Aminco). Chlorophyll a ( $\mathrm{chl}$ a) and carotenoid concentrations were calculated (Lichtenthaler 1987). Cell number was counted with a haemocytometer. Biomass dry weight (DW) was estimated by filtering a known volume of culture through a pre-dried and preweighed glass fibre filter (GF/C, Whatman) and then drying the filter for $1 \mathrm{~h}$ at $110^{\circ} \mathrm{C}$.

Other biochemical parameters. Absorption coefficients of the cells were measured using the Shibata technique (Shibata et al. 1954) with standard white A4 copy paper as a light diffuser and a UVmin 1240 visible UV light (UV/VIS) spectrophotometer (Shimadzu). Absorption $\left(\mathrm{cm}^{-1}\right)$ was divided by the chl a concentration, converted to natural log values and expressed per meter to obtain the standard optical cross sections $\left(\mathrm{m}^{2}\right.$ $\mathrm{mg}^{-1} \mathrm{chl}$ a).

Incident solar radiation was measured with a pyranometer (Li-Cor) every $5 \mathrm{~min}$ and stored in a data logger (Li-Cor 1000) assuming that $1 \mathrm{~W} \mathrm{~m}^{-2}$ equals $4.5 \mu \mathrm{mol}$ photons $\mathrm{m}^{-2} \mathrm{~s}^{-1}$ and that photosynthetically active radiation (PAR) is $40 \%$ of the total solar irradiance.

Photosynthetic measurements. Dual pulse amplitude modulation (PAM): The dual PAM fluorometer (Walz) measures the Photosystem II (PSII) efficiency simultaneously with the absorption changes of Photosystem I (PSI) using the pulse modulation principle in the dual wavelength approach (Klughammer \& Schreiber 2008). We used this instrument to carry out rapid light curves (RLC) of photosynthetic electron transport rates as a function of irradiance. Samples were concentrated $\sim 10$-fold by centrifugation ( $6 \mathrm{~min}$ at $2900 \times g$ ). Sample handling was performed in dim light within 15 to 20 min, which means that all RLCs were performed with the dark-adapted samples, and that the measured RLCs were likely to measure potential photosynthetic activity (White \& Critchley 1999). Three replicate RLCs were measured under increasing actinic light intensities lasting $30 \mathrm{~s}$ each. PSII electron transport rates (ETR) were calculated as relative rates by multiplying the irradiance $(E)$ with the effective quantum efficiency $\left(\Delta F / F_{\mathrm{m}}{ }^{\prime}\right)$, i.e. $\left.\mathrm{rETR}=E \times \Delta F / F_{\mathrm{m}}{ }^{\prime}\right)$. $\Delta F$ equals the maximum fluorescence measured at irradiance $E\left(F_{\mathrm{m}}{ }^{\prime}\right)$ minus the steady state fluorescennce $F$. All RLCs were fitted using the exponential model of Webb et al. (1974).

We also measured RLCs on sub-samples immediately withdrawn from the 2 culture systems using the water pulse amplitude modulation, water PAM (Walz). As such, and in contrast to the dual PAM data, these samples had not been dark adapted before RLC measurements.

Flow-through water PAM: This is a highly sensitive chlorophyll fluorometer designed for continuously monitoring photosynthetic activity of phytoplankton and algal suspensions. It consists of a waterproof measuring head covered by a flow-through chamber and is operated in conjunction with the standard PAM control unit via a personal computer with the Win Control software (Walz). The chamber is connected to a peristaltic pump, which continuously pumped samples from the algal photobioreactor into the waterproof measuring head. Simulated in situ RLCs were acquired by repeated measurements of fluorescence responses to saturating flashes, followed by a $30 \mathrm{~s}$ illumination period of actinic light at increasing intensities. This procedure provided a series of photosynthetic fluorescence yields (PFYs) as a function of the actinic light intensity. Maximum PFY in the light-adapted state measured with the flow-through water PAM was calculated as: $\left(F_{\mathrm{m}}{ }^{\prime}-F_{\mathrm{o}}{ }^{\prime}\right) / F_{\mathrm{m}}{ }^{\prime}=F_{\mathrm{v}}{ }^{\prime} / F_{\mathrm{m}}{ }^{\prime}$, where $F_{\mathrm{v}}{ }^{\prime} / F_{\mathrm{m}}{ }^{\prime}$ is the maximum efficiency of PSII and $F_{\mathrm{o}}{ }^{\prime}$ is the minimal fluorescence in the light-adapted state. Three replicates RLC were measured for both production systems on each sampling time. On several occasions, in situ variations in PFY were followed by a series of repetitive saturating pulses separated from each other by 2 to $3 \mathrm{~min}$.

Fluorescence induction curves: A portable fluorometer, the Aquapen AP 100 (Photon System Instruments), fitted with red light-emitting diodes (LEDs) to provide saturating light intensity was used to follow the polyphasic rise of chl a fluorescence in both outdoor cultures, the so-called Kautsky curve. Darkadapted (10 min) samples were diluted to a final concentration of about $200 \mathrm{mg}$ biomass $\mathrm{l}^{-1}$ (about $3.5 \mathrm{mg}$ $\mathrm{chl} \mathrm{l}^{-1}$ ) and were placed in a $3 \mathrm{ml}$ fluorescence cuvette, which was mounted in front of the detector while the illuminating red LEDs supplied high intensity light from both sides, perpendicular to the detector. Rapid fluorescence induction curves were recorded in the time range between $50 \mu$ s and $2 \mathrm{~s}$ from the onset of the saturation light. At least 3 curves were recorded for each sample and averaged. The fluorescence induction curve rises from the minimum fluorescence $F_{\mathrm{o}}(\mathrm{O})$ to the maximum fluorescence $F_{\mathrm{m}}(\mathrm{P})$ via 2 inflection 
points, $\mathrm{J}$ and I, resulting in the well-known OIJP transient, where $\mathrm{O}, \mathrm{J}, \mathrm{I}$ and $\mathrm{P}$ are inflection points on the fluorescence induction curve (e.g. Strasser et al. 1995). $V_{j}$ or $V_{i}$ are the chlorophyll fluorescence yields at the corresponding points on the fluorescence induction curve. From the fluorescence levels at J $\left(F_{j}\right)$ and I $\left(F_{i}\right)$ the parameters $V_{j}$ and $V_{i}$ were calculated as follows:

$$
V_{j}=\left(F_{j}-F_{\mathrm{o}}\right) /\left(F_{\mathrm{m}}-F_{\mathrm{o}}\right) \text { and } V_{i}=\left(F_{i}-F_{\mathrm{o}}\right) /\left(F_{\mathrm{m}}-F_{\mathrm{o}}\right)
$$

Photosynthetic oxygen evolution: Photosynthesis and respiration rates in response to an irradiance level were measured as oxygen exchange with a Clark-type polarographic oxygen electrode (model 5331, Yellow Springs Instruments) while the illumination of the algal sample was provided by a light pipette (Brammer, Illuminova) as described by Dubinsky et al. (1987) and measured by a LI-250A light meter connected to LI-190 Quantum Sensor (Li COR). Samples collected from the HRAP or FPP were diluted twice with a fresh medium, placed into the incubation chamber (ca. $15 \mathrm{ml}$ ) and equilibrated to the chamber temperature $\left(25^{\circ} \mathrm{C}\right)$ in the dark. After 20 min of dark incubation, the respiration rate was measured for $120 \mathrm{~s}$. Net photosynthetic rates were measured while the incubation chamber was illuminated with a sequence of 9 irradiances $(6,44,80$, $130,166,280,350,580$ and $740 \mu \mathrm{mol}$ photons $\mathrm{m}^{-2} \mathrm{~s}^{-1}$ ) provided by the light pipette. Each irradiance period lasted for $120 \mathrm{~s}$. The experiments were duplicated in most cases. The calculations of the photosynthetic rate were carried out with the average values of gross oxygen evolution during the light period except for the first few seconds of the transition between conditions. The $\mathrm{P}$ versus $\mathrm{E}$ equations and parameters $\left(\alpha, P_{\max }, E_{\mathrm{k}}\right.$ and $E_{\text {com }}$ ) were fitted using a hyperbolic tangent model (Platt \& Jassby 1976).

\section{RESULTS}

\section{Irradiance, temperature and $\mathrm{pH}$}

Changes in the physico-chemical environment in the 2 culture systems are shown in Fig. 2 (pH and temperature) and Fig. 3 (incident photon flux). Incident photon flux during the experiment was similar on all $3 \mathrm{~d}$ of the experiment, attaining maximal values of $\sim 2000 \mu \mathrm{mol}$ photons $\mathrm{m}^{-2} \mathrm{~s}^{-1}$ at around 13:00 h (Fig. 3). In concert with the increasing solar irradiance, the temperature in both systems increased during the day, peaking after 15:00 h. However, daily changes in temperature were not as marked for the HRAP as they were for the FPP (Fig. 2), which reached a high supraoptimum value of $41^{\circ} \mathrm{C}$ on 3 April when the cooling capacity was insufficient. Similarly, the $\mathrm{pH}$ changes of the culture were not as dramatic in the HRAP; they

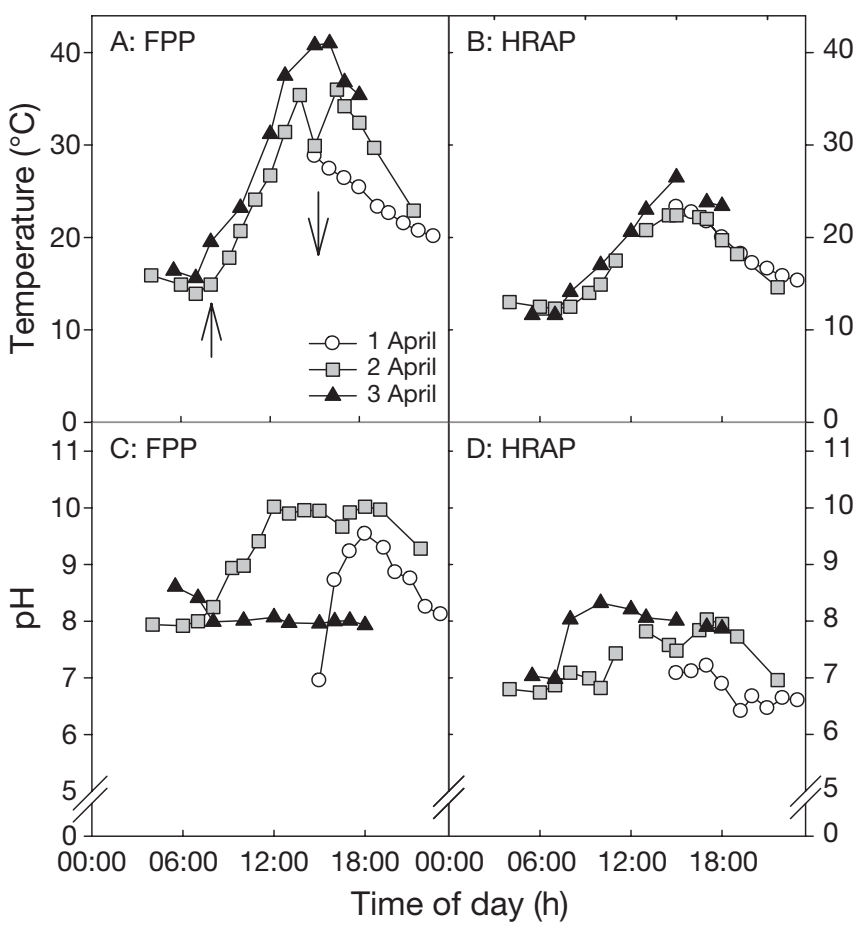

Fig. 2. Changes in $(A, B)$ temperature and $(C, D) p H$ for the flat panel photobioreactor $(\mathrm{FPP} ; \mathrm{A}, \mathrm{C})$ and the high rate algal pond (HRAP; $\mathrm{B}, \mathrm{D})$. Changes in operation of the FPP $\left(\mathrm{CO}_{2}\right.$ on/off on 3 April) are indicated by the arrows

increased from $\mathrm{pH} 7$ in the morning to $\mathrm{pH} 8.3$ in the afternoon, and returned to $\mathrm{pH} 7$ in the late evening. However, without $\mathrm{CO}_{2}$ control, as was exercised on Day 2, pH rose considerably in the FPP, attaining values of 10 whereas on Day 3 the pH was kept constant at $\mathrm{pH} 8$ (Fig. 2).

Although we could not measure the diffuse light attenuation coefficient $\left(k_{\mathrm{d}}\right)$ we tried to estimate the

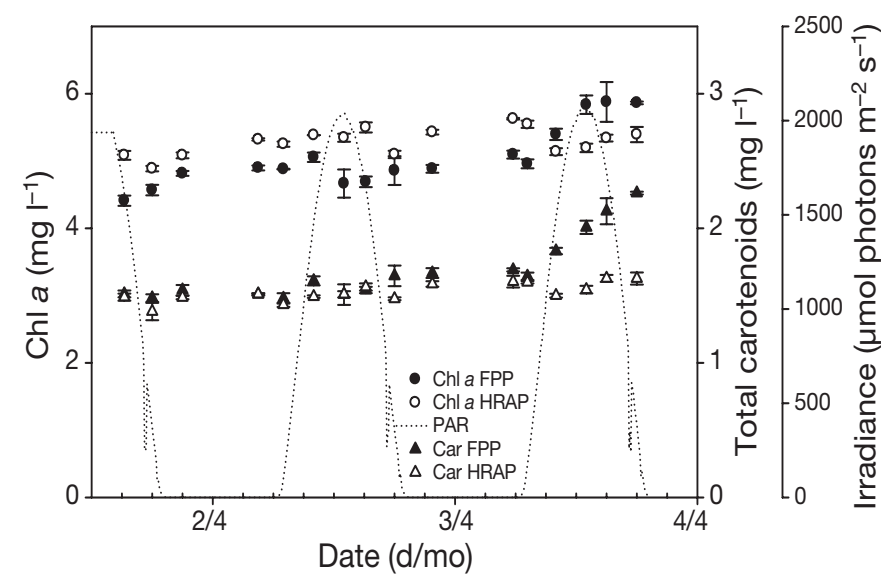

Fig. 3. Changes in chl a concentrations (O) and total carotenoids $(\Delta)$ in the FPP (closed symbols) and HRAP (open symbols), 2008. The dotted line represents the incident irradiance 
average irradiance in the HRAP and FPP. From a mass culture of Nannochloropsis with a chl a content per cell similar to that of the HRAP culture, a $k_{\mathrm{d}}$ of $66.5 \mathrm{~m}^{-1}$ was measured at a cell density of $57 \times 10^{6} \mathrm{cells} \mathrm{ml}^{-1}(\mathrm{~J}$. C. Kromkamp \& F. van Hoogstraten unpubl.). Using the data from Table 1 we calculated $k_{\mathrm{d}}$ values of 51.4 and $124 \mathrm{~m}^{-1}$ in the HRAP and FPP, respectively. For the FPP culture this might be an overestimation because of the lower cellular chl a content. As the daily average irradiance was $1130 \mu \mathrm{mol}$ photons $\mathrm{m}^{-2} \mathrm{~s}^{-1}$ we calculated an average daily irradiance of $93 \mu \mathrm{mol}$ photons $\mathrm{m}^{-2} \mathrm{~s}^{-1}$ in the HRAP and $222 \mu \mathrm{mol}$ photons $\mathrm{m}^{-2} \mathrm{~s}^{-1}$ in the FPP. This calculation was done assuming a path length of $35 \mathrm{~cm}$ for the HRAP and $5 \mathrm{~cm}$ for the FPP. These path lengths might increase with changing sun angle. However, because the cell densities were so high, a diffuse light gradient will develop quickly making the angle of the sun less important.

\section{Light climate, biomass and pigmentation}

Both cultures showed an increase in the chl a concentration with time (Fig. 3). According to the National Mariculture Center (NCM), on which premises we performed our experiments, the HRAP had a dilution rate of $0.1 \mathrm{~d}^{-1}$. From this and the change in chl a concentration we calculated the specific growth rate in this culture during the measuring period as $0.13 \pm 0.013 \mathrm{~d}^{-1}$ (mean $\pm \mathrm{SE}$ ). How precise this value is depends on the accuracy of the water addition to compensate for the evaporation.

The FPP showed a higher daily rate of increase in chl a (Fig. 3), but this was partly due to the fact that on Day 1 and Day 3 there was no dilution in the system

Table 1. Average $( \pm \mathrm{SE})$ dry weight $(\mathrm{DW})$, cell number and pigment content per culture volume during Day 3

\begin{tabular}{|lccccc|}
\hline $\begin{array}{l}\text { Culture } \\
\text { system }\end{array}$ & $\begin{array}{c}\text { Carotenoids } \\
\left(\mathrm{mg} \mathrm{l}^{-1}\right)\end{array}$ & $\begin{array}{c}\mathrm{Chl} \mathrm{a} \\
\left(\mathrm{mg} \mathrm{l}^{-1}\right)\end{array}$ & $\begin{array}{c}\text { Cells } \\
\left(\times 10^{6} \mathrm{ml}^{-1}\right)\end{array}$ & $\begin{array}{c}\mathrm{DW} \\
\left(\mathrm{mg} \mathrm{l}^{-1}\right)\end{array}$ & $\begin{array}{c}\text { Carotenoids: } \\
\text { chl } a \text { ratio }\end{array}$ \\
\hline FPP & $1.7 \pm 0.24$ & $5.1 \pm 0.46$ & $82 \pm 13.8$ & $561 \pm 36.5$ & $0.32 \pm 0.02$ \\
HRAP & $1.5 \pm 0.07$ & $5.3 \pm 0.20$ & $44 \pm 4.8$ & $229 \pm 21.8$ & $0.27 \pm 0.01$ \\
\hline
\end{tabular}

Table 2. Average $( \pm \mathrm{SE})$ dry weight (DW), cell number and pigment content per biomass unit (DW or cell) during the $3 \mathrm{~d}$ measuring period

\begin{tabular}{|lcccc|}
\hline $\begin{array}{l}\text { Culture } \\
\text { system }\end{array}$ & $\begin{array}{c}\mathrm{Chl} \mathrm{a} \\
\left(\mathrm{fg} \mathrm{cell}^{-1}\right)\end{array}$ & $\begin{array}{c}\text { DW } \\
\left(\mathrm{pg} \mathrm{cell}^{-1}\right)\end{array}$ & $\begin{array}{c}\text { Chl a } \\
\left(\mathrm{mg} \mathrm{g}^{-1} \mathrm{DW}\right)\end{array}$ & $\begin{array}{c}\text { Carotenoids } \\
\left(\mathrm{fg} \mathrm{cell}^{-1}\right)\end{array}$ \\
\hline FPP & $60 \pm 6.1$ & $6.7 \pm 0.89$ & $9 \pm 1.0$ & $20 \pm 2.8$ \\
HRAP & $121 \pm 13.3$ & $5.2 \pm 0.73$ & $23 \pm 2.3$ & $35 \pm 4.4$ \\
\hline
\end{tabular}

and the calculated growth rate for those periods equalled approximately $0.20 \mathrm{~d}^{-1}$. On Day 2 , the culture was diluted by $\sim 15 \%$ accounting for the smaller absolute changes in chl a $\mathrm{l}^{-1}$ shown in Fig. 3 at this time. The overall growth rate in the FPP equalled $0.17 \pm 0.019 \mathrm{~d}^{-1}$. As the $95 \%$ confidence intervals overlapped, we could thus not conclude that these growth rates were significantly different.

DW and cell number showed more variation than the chl a content and did not show a significant change during the $3 \mathrm{~d}$ measuring period (Tables 1 \& 2). Only the slight DW increase in the HRAP was significant (ANOVA, p < 0.01). Although the chl a concentrations hardly differed between both systems, the DW of the FPP was much higher $\left(0.56 \mathrm{~g} \mathrm{l}^{-1}\right)$ than that of the HRAP $\left(0.23 \mathrm{~g} \mathrm{l}^{-1}\right)$. The cell concentrations were also twice as high in the FPP than in the HRAP. As a result the chl a/DW (w/w) ratio was 2.5 times higher in the HRAP than in the FPP (Table 2). This result suggests a greater shade-adaptation by the HRAP culture compared with the FPP culture, reflecting the greater degree of light attenuation in the deeper culture and consequent longer optical light path. The DW per cell in the FPP was $32 \%$ higher than that in the HRAP. We do not know whether this is due to a higher concentration of protein, fatty acids/lipids or carbohydrates. The maximal photosynthetic rates per cell based on oxygen exchange (but not per unit chlorophyll, see Fig. 7) were higher in the FPP than in the HRAP, suggesting that Rubisco levels (and hence protein) might have been higher in the former cultures.

Total carotenoids were about $15 \%$ higher in the FPP than in the HRAP, and the carotenoids/chl a ratio was also higher ( 0.32 and 0.27 for the FPP and HRAP, respectively). However, the total carotenoids per cell were $75 \%$ higher in the HRAP cells than in the FPP cells, in accordance with its shade-acclimated pattern. Because no HPLC data were available we cannot comment on the composition of the light-harvesting versus the protective carotenoids. On Day 3, the carotenoids showed a marked increase in the FPP, most probably as a result of the stressful conditions imposed by the suboptimal temperatures during that day (Fig. 2). Also the carotenoid/chl a ratio increased from 0.33 to 0.40 , indicating a stress response.

\section{Absorption cross section}

The optical absorption cross section $\left(\mathrm{a}^{*}\right)$ of the Nannochlorpsis cells in the 
FPP decreased from $0.0090 \mathrm{~m}^{2} \mathrm{mg}^{-1} \mathrm{chl} \mathrm{a}$ measured in the evening of 1 April to $0.0077 \mathrm{~m}^{2} \mathrm{mg}^{-1} \mathrm{chl} a$ at 10:00 h on Day 3 . This is higher than the $\mathrm{a}^{*}$ of $0.0068 \mathrm{~m}^{2}$ $\mathrm{mg}^{-1} \mathrm{chl} a$ of the algal cells in the HRAP (Table 3). This was expected because the HRAP cells had a higher chl a content per cell. Hence, the shade adaptation pattern of the HRAP cells caused a higher degree of pigment packaging. Interestingly, on 3 April $\mathrm{a}^{*}$ of the FPP cells increased from 0.0077 at $10: 00 \mathrm{~h}$ to $0.0082 \mathrm{~m}^{2} \mathrm{mg}^{-1} \mathrm{chl}$ a several hours later. Most probably, this was due to the induction of carotenoid synthesis on this day (which increased from 1.14 at 07:00 h to $1.39 \mathrm{mg} \mathrm{l}^{-1}$ at 13:00 h). These values of $\mathrm{a}^{*}$ are at the low end of the range of natural variability, reflecting the cultured organism's already small size of $\mathrm{a}^{*}$ and probably low degree of packaging.

\section{Photosynthetic performance}

Photosynthetic characteristics measured by the water PAM and the dual PAM

For both the water PAM and the dual PAM measurements, we observed that the culture in the HRAP had significantly lower relative photosynthetic electron transport $\left(\mathrm{rETR}_{\max }\right)$ values than those in the FPP (Fig. 4). The HRAP culture also showed less variation during the experimental period. Such reductions in maximal rates of photosynthesis are consistent with a greater degree of shade adaptation in the HRAP (Richardson et al. 1983). The $\mathrm{rETR}_{\max }$ in the FPP was strongly depressed during the afternoon on Day 2, possibly due to $\mathrm{CO}_{2}$ depletion. This was both visible with and without dark acclimation, although the water PAM data (i.e. no dark acclimation) showed a much larger

Table 3. Absorption cross sections $\left(\mathrm{m}^{2} \mathrm{mg}^{-1} \mathrm{chl}\right.$ a) of the Nannochloropsis cultures in the 2 mass culture systems. nd: no data. FFP: flat panel photobioreactor; HRAP: high rate algal pond

\begin{tabular}{|lccc|}
\hline Date & Time of day (h) & FPP & HRAP \\
\hline 1 April 08 & $20: 00$ & 0.0090 & 0.006 .7 \\
3 April 08 & $10: 00$ & 0.0077 & nd \\
3 April 08 & $12: 00$ & 0.0082 & 0.0068 \\
\hline
\end{tabular}

decrease in $\mathrm{rETR}_{\max }$. The high irradiance was not the main factor for the depression, which can be deduced from the comparison between the 16:00 h samples on Day 1 (e.g. water PAM: $\mathrm{rETR}_{\max }=155$ relative units [r.u.]) and Day 2 (rETR ${ }_{\max }=72$ r.u.). The $\mathrm{pH}$ stat experiment on Day 3, in which we kept the pH constant, was paralleled by the supra-optimum temperature (see accompanying paper by Sukenik et al. 2009). This excessively high temperature clearly induced an even larger decrease in $\mathrm{rETR}_{\max }$ measured by both techniques at mid-day than observed the previous day. In any case, at night the difference in $\mathrm{rETR}_{\max }$ between the 2 cultures was diminished and according to expectations both water PAM and dual PAM measured similar rETR max $_{\text {val- }}$ ues (except in the early night on 3 April in the HRAP). Also, the values of $\mathrm{rETR}_{\max }$ at night are relatively low due to the well-known downregulation of enzyme activity during the darkness, which was especially strong 
in the FPP culture (Fig. 4A). Note that the water PAMderived $\mathrm{rETR}_{\max }$ values were higher during the morning and evening periods than were the dual PAM-derived $\mathrm{ETR}_{\max }$ values, whereas at night they were similar. This suggests that downregulation of enzyme activity already started during the 20 to 30 min dark acclimation time used for the dual PAM measurements.

Water PAM alpha values based on the RLC measurements were significantly decreased around midday/early afternoon in both FPP and HRAP cultures (Fig. 4). The variability in the dual PAM $\alpha_{\text {ETR }}$ values was much less pronounced. However, in contrast to the $\mathrm{rETR}_{\max }$ values, $\alpha_{\mathrm{ETR}}$ of the water PAM samples was not different between the 2 culture systems (ANOVA, $\mathrm{p}=0.63$ ). Although the average values measured after dark adaptation (dual PAM) of the FPP and HRAP values were similar ( 0.73 and 0.79 , respectively) the small difference was significant (ANOVA, p < 0.01). Comparing the nightly $\alpha_{\mathrm{ETR}}$ values between the dual PAM and water PAM is difficult because the values depend on the accuracy of the calibration of the internal light sources of the PAMs. As we could not perform an intercalibration we cannot say whether the differences measured during the night were significant. However, the increase in $\alpha_{\text {ETR }}$ during the 2nd night measured with the water PAM was not evident with the dual PAM. At present we cannot explain this.

The light saturation parameter $E_{\mathrm{k}}\left(=\mathrm{rETR} \mathrm{R}_{\mathrm{max}} / \alpha_{\mathrm{ETR}}\right)$ of the FPP was substantially higher than $E_{\mathrm{k}}$ of the cells in the HRAP. This was observed both for the water PAM measurements (values averaged 466 versus $264 \mu \mathrm{mol}$ photons $\mathrm{m}^{-2} \mathrm{~s}^{-1}$ for the FPP and HRAP, respectively) and the dual PAM measurements (261 versus $162 \mu \mathrm{mol}$ photons $\mathrm{m}^{-2} \mathrm{~s}^{-1}$ for the FPP and HRAP, respectively). This again clearly demonstrates shade adaptation of the HRAP cells and light acclimation of the FPP cells.

\section{Dual PAM $F_{\mathrm{v}} / F_{\mathrm{m}}$ data}

The maximum PSII quantum efficiency $\left(F_{\mathrm{v}} / F_{\mathrm{m}}\right)$ measured after 15 to 20 min of darkness adaptation clearly revealed a decrease at early afternoon followed by a recovery in the evening (Fig. 5); between 18:00 and 19:30 h, $F_{\mathrm{v}} / F_{\mathrm{m}}$ fully recovered. It can be expected that dynamic downregulation of non-photochemical quenching (NPQ) will relax if it is due to the xanthophyll cycle only. This was not the case and this suggests the existence of long-lived quenchers, most probably caused by photodamage to PSII, corroborated by the observed PSII heterogeneity or $\mathrm{Q}_{\mathrm{B}}$-non-reducing PSII centres (see next section for explanation). On the first $2 \mathrm{~d}$ of the experiments the difference in $F_{\mathrm{v}} / F_{\mathrm{m}}$ between the FPP and HRAP samples was not significant, suggesting the $\mathrm{CO}_{2}$ depletion in the FPP on Day 2

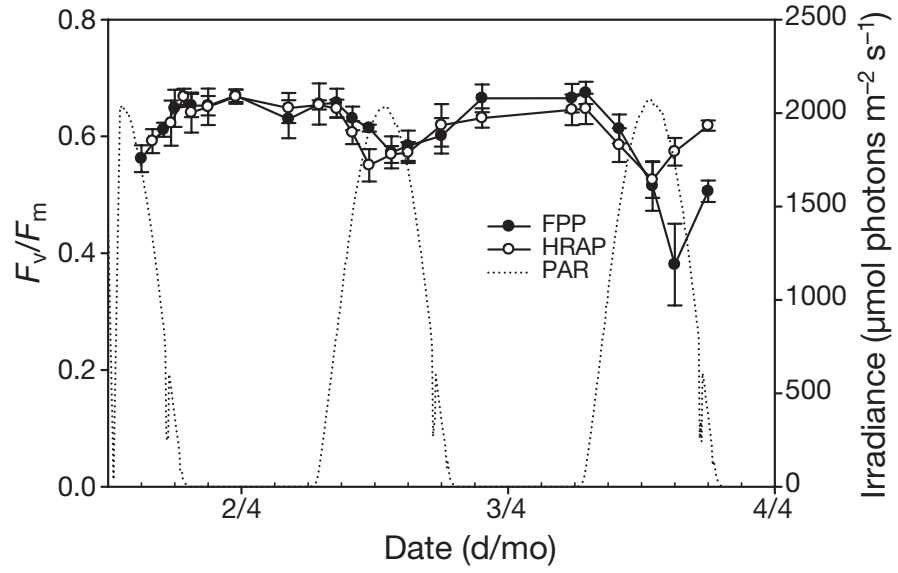

Fig. 5. $F_{\mathrm{v}} / F_{\mathrm{m}}$ values of the flat panel photobioreactor (FPP) and high rate algal pond (HRAP) measured during the experimental period in 2008 using the dual pulse amplitude modulation

(PAM). Incident irradiance is plotted as a dotted line

did not influence $F_{\mathrm{v}} / F_{\mathrm{m}}$. However, the rise in temperature on Day 3 in the FPP caused a large decrease in $F_{\mathrm{v}} / F_{\mathrm{m}}(0.38)$, although at 18:00 h $F_{\mathrm{v}} / F_{\mathrm{m}}$ showed a trend of recovery $(0.58)$.

\section{The chl a fluorescence induction kinetics}

The rapid chl a fluorescence induction kinetics of all oxygenic photosynthetic organisms show a polyphasic rise (chl a fluorescence transient) between the initial $\left(F_{\mathrm{o}}\right)$ and the maximum $\left(F_{\mathrm{m}}\right)$ fluorescence during the first second of illumination (Neubauer \& Schreiber 1987). These phases have been designated as O, J, I and $\mathrm{P}$, and can be visualized using a logarithmic time scale (Strasser et al. 1995). The current understanding of the OJIP transient rise is that it reflects the filling up (i.e. reduction) of the electron acceptor pool (phaeophytine (Ph), $\mathrm{Q}_{\mathrm{A}}, \mathrm{Q}_{\mathrm{B}}$ and PQ pool) of PSII in a 4-photon process (Strasser et al. 2004, Lazár 2006). The inflection $\mathrm{J}(2 \mathrm{~ms}$ ) represents the double reduction of electron carriers $\mathrm{Ph}, \mathrm{Q}_{\mathrm{A}}$ and $\mathrm{Q}_{\mathrm{B}}$, and, perhaps because of a limitation in electron acceptance by $Q_{B}$, this step usually occurs when cells are exposed to excessive light that increases the degree of reduction of the PQ pool, while the I-step (30 ms) is connected to a 3-electron reduction in the PSII electron carriers to different redox states (e.g. $\mathrm{Ph}_{\mathrm{A}}{ }^{-} \mathrm{Q}_{\mathrm{B}}{ }^{2-}, \mathrm{Ph}^{-} \mathrm{Q}_{\mathrm{A}} \mathrm{Q}_{\mathrm{B}}{ }^{2-}$ or $\mathrm{Ph}^{-} \mathrm{Q}_{\mathrm{A}}^{-}$ $Q_{B}$ ) of the reaction centre complex, which reduces the PQ pool (Lazár 2006). The I-step probably reflects the heterogeneity of the PQ pool, with fast-reducing and slow-reducing PSII centres. Hence, slow reaction centres may provide a mechanism for quenching excessive energy (Strasser et al. 1995). The drop in the induction curve of microalgal cells beyond the P-step indicates that the PQ pool is being re-oxidized due to 
the demand of reducing equivalents from the CalvinBenson cycle. Thus, the 'JIP' test can be used as a rapid monitor of photosynthetic activity, including the effects of various stressors on this process.

The Aquapen AP100 was applied to record fluorescence induction curves in Nannochloropsis samples collected from the FPP and HRAP on $3 \mathrm{~d}$ of measurements.

The induction curve kinetics measured on Day 1 showed little difference between the parameters of the HRAP and FPP (Fig. 6). On Day 2, when $\mathrm{CO}_{2}$ depletion occurred, the FPP culture was more photo-stressed than on the previous day or in the HRAP culture: between 13:00 and 15:00 $\mathrm{h}$ over-reduction of the PSII acceptors was observed as could be inferred from higher $V_{j}$ and $V_{i}$ values. The $F_{\mathrm{v}} / F_{\mathrm{m}}$ values were also more reduced than the on previous days, as was also observed in the dual PAM data.

Striking discrepancies between the FPP and HRAP cultures were found on Day 3 when the temperature in the FPP temporarily rose to $41^{\circ} \mathrm{C}$. This caused significant over-reduction of the reducing side of the PSII complex: the $V_{j}$ and $V_{i}$ parameters did not recover in the afternoon. This was accompanied by a dramatic depression of $F_{\mathrm{v}} / F_{\mathrm{m}}$ in the FPP culture compared with the HRAP culture (Fig. 6C,F). These results suggest that high temperature stress was not released and the $\mathrm{Q}_{\mathrm{B}}$-non-reducing centres persisted. The time course of $V_{j}, V_{i}$ and $F_{\mathrm{v}} / F_{\mathrm{m}}$ parameters on both days showed that photoinhibitory stress was strongest at 15:00 h, most

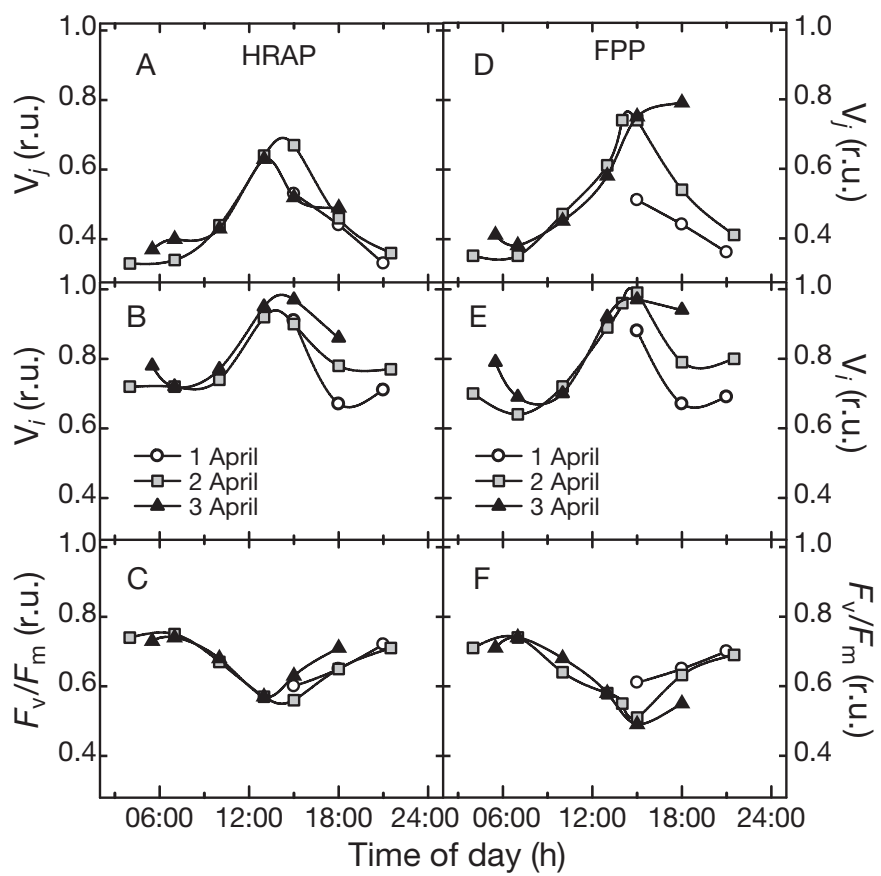

Fig. 6. Daily course of fluorescence parameters $V_{j,} V_{i}$ and $F_{\mathrm{v}} / F_{\mathrm{m}}$ measured on Days 1 to 3 in the Nannochloropsis cultures grown in the (A-C) high rate algal pond (HRAP) and (D-F) flat panel photobioreactor (FPP) probably induced by culture overheating. This observation can be attributed to a continuous damage to the D1 protein, whose repair is retarded due to photoinhibition during the early afternoon hours (Takahashi \& Murata 2008, Zhao et al. 2008).

\section{Oxygen exchange}

\section{Photosynthetic parameters from oxygen evolution}

Patterns in the maximum rate of oxygen evolution $\left(P_{\max }\right)$ and the initial slope $(\alpha)$ of the photosynthesis irradiance (PE) curve were not as clear as those in rETR (Fig. 7). The algal cells in the HRAP showed a decrease $\alpha$, whereas $P_{\max }$ increased from approximately 150 to $300 \mu \mathrm{mol} \mathrm{O} \mathrm{Og}^{-1}$ (chl a) $\mathrm{h}^{-1}$ from the start of the experiment to the morning of Day 3, after which it remained more or less constant (Fig. 7A). The $\alpha$ variable in the FPP culture showed a considerable amount of scatter, but did not seem to change significantly during the first $2 \mathrm{~d}$ of the experiment (Fig. 7D). However, on Day 3, $\alpha$ decreased considerably during the day and $P_{\max }$ showed a similar pattern, suggesting a closer coupling between $P_{\max }$ and $\alpha$ of Nannochloropsis in the FPP than in the HRAP. The $P_{\max }$ values were higher in the FPP culture than that of the HRAP (ANOVA, $p=0.001$ ). The $\alpha$ values of the FPP were slightly higher than those of the HRAP (ANOVA, $\mathrm{p}=0.02$ ) Algal respiration in the HRAP was significantly lower (ANOVA, $p<0.01$ ) than in the FPP. The patterns in respiration were mirrored by the variability in the compensation irradiance $E_{\mathrm{c}}$. Although considerable variability was observed in both parameters a clear trend was absent. An increase during the light period in dark respiration and in $E_{c}$ values was observed in the HRAP (Fig. 7B). The pattern was less clear in the FPP: a minimum in both $E_{\mathrm{k}}$ and $E_{\mathrm{c}}$ seem to be present during the middle of the light period (Fig. 7E). The light saturation parameter $E_{\mathrm{k}}\left(=P_{\max } / \alpha\right)$ of the HRAP culture increased during the first $2 \mathrm{~d}$ of the experiment until it reached the stable value on Day 3, similar to those of the FPP, which did not show any significant trend. In contrast to the $E_{\mathrm{k}}$ values of the ETR/E curves, the differences in $E_{\mathrm{k}}$ based on the $\mathrm{O}_{2}$ exchange data were only slightly higher in the FPP than those of the HRAP (ANOVA, $p=0.04$ ).

\section{Quantum efficiencies of PSII charge separation and} oxygen evolution

To compare the efficiency of the photosynthetic process we calculated the quantum efficiencies of PSII charge separation $\left(\Phi_{\mathrm{P}}\right)$ and of oxygen evolution $\left(\Phi_{\mathrm{O} 2}\right)$ 

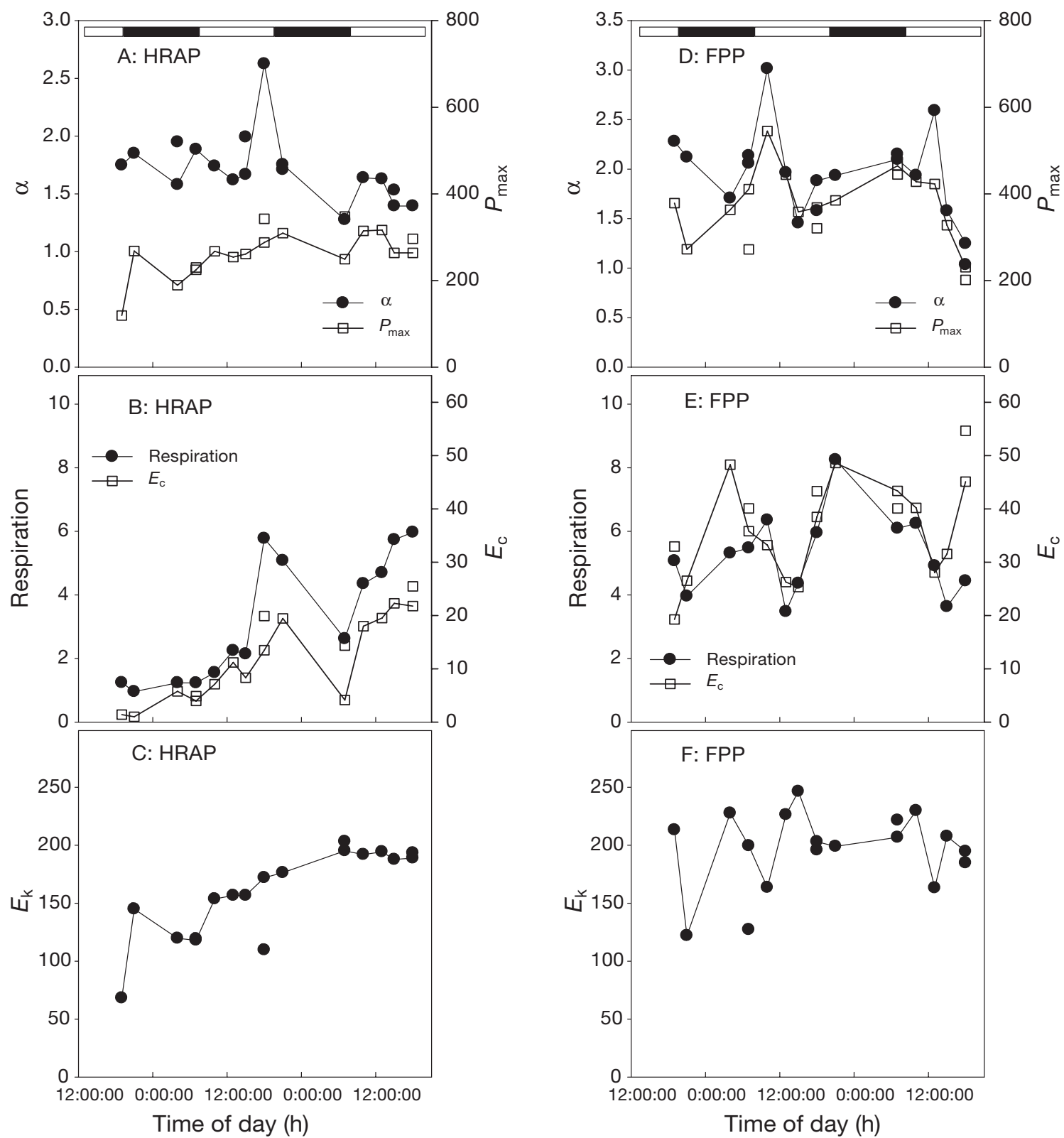

Fig. 7. Photosynthetic parameters, as determined by oxygen exchange measurements, of Nannochloropsis grown in the high rate algal pond (HRAP) (A-C) or flat panel photobioreactor (FPP) (D-F). $P_{\max }: \mu$ mol O $\mathrm{O}_{2} \mathrm{mg}(\mathrm{chl} a)^{-1} \mathrm{~h}^{-1}$; respiration: $\mathrm{nmol} \mathrm{O}_{2}$ cell $^{-1} \mathrm{~h}^{-1}$; $E_{\mathrm{c}} E_{\mathrm{k}}$ : $\mu \mathrm{mol}$ photons $\mathrm{m}^{-2} \mathrm{~s}^{-1} ; \alpha$ : $\mu \mathrm{mol} \mathrm{O} \mathrm{O}_{2}(\mathrm{mg} \mathrm{chl} \mathrm{a})^{-1} \mathrm{~h}^{-1}$ ( $\mu \mathrm{mol}$ photons $\left.\mathrm{m}^{-2} \mathrm{~s}^{-1}\right)^{-1}$. The day/night period is given at the top of the panels as a light/dark bar

as follows, ignoring spectral differences between the light sources, which might influence the data:

$$
\Phi=P_{\max } /\left(\mathrm{a}^{*} \times E\right),
$$

where $P_{\max }$ is the maximum rate of oxygen evolution or absolute ETR. Absolute ETR was calculated as:

$$
\mathrm{ETR}=E \times \Delta F / F_{\mathrm{m}}{ }^{\prime} \times \mathrm{a}^{*} \times F_{\mathrm{II}}
$$

where $F_{\text {II }}$ is the ratio of the optical absorption cross section of PSII relative to the total absorption cross section
(Kromkamp \& Forster 2003); it is often assumed that both the PSI and PSII antenna absorb $50 \%$ of the light (Gilbert et al. 2000). According to Suggett et al. (2004) $F_{\text {II }}$ varies between 0.5 and 0.6 for most taxa, and for the purpose of our calculations we assumed that $F_{\text {II }}$ equalled 0.5. Subsequently, we plotted $\Phi_{\mathrm{O} 2}$ as a function of $\Phi_{\mathrm{P}}$ of the HRAP cells (Fig. 8). The plots show a linear relationship between both quantum efficiencies, demonstrating a tight coupling between the different processes in the light reactions of photosynthesis. The 


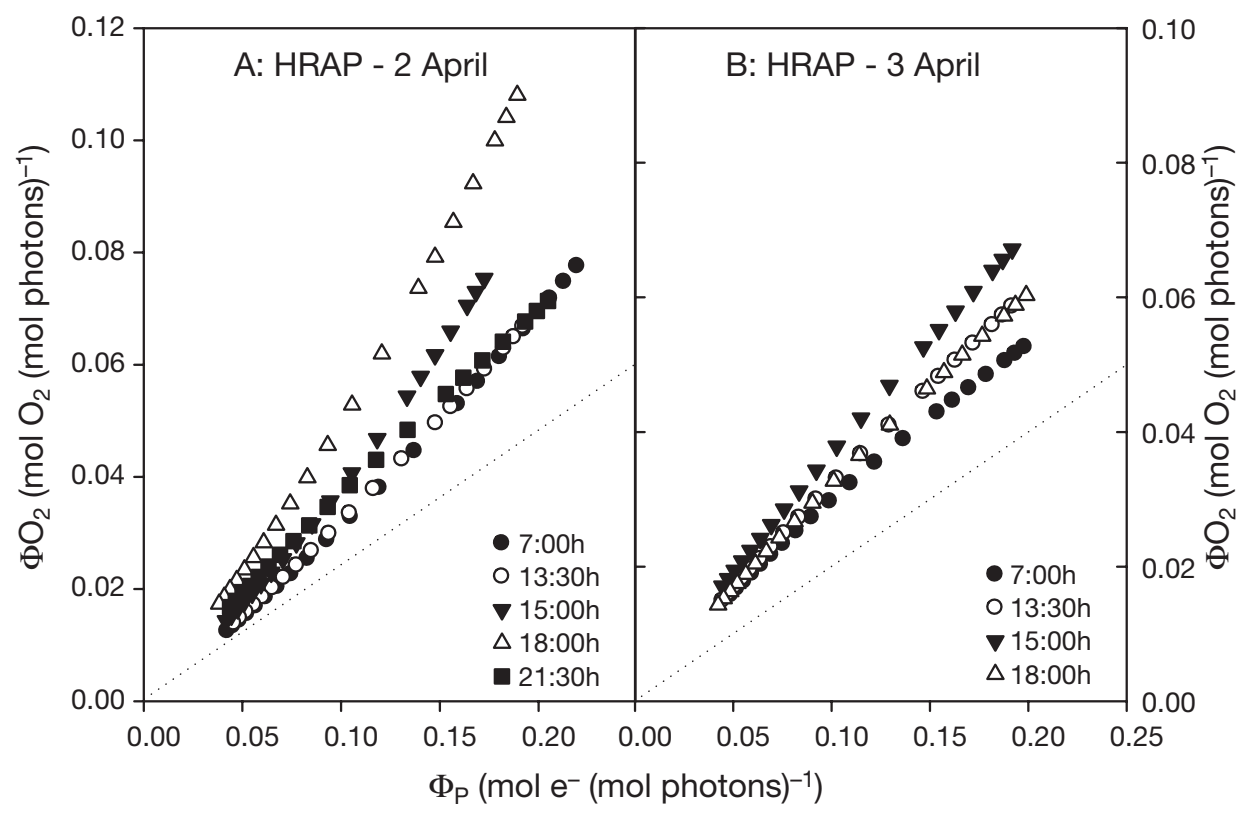

Fig. 8. Quantum efficiencies of oxygen evolution $\left(\Phi \mathrm{O}_{2}\right)$ plotted as a function of the quantum efficiencies of Photosystem II charge separation $\left(\Phi_{\mathrm{P}}\right)$ of the Nannochloropsis cells in the high rate algal pond (HRAP) on 2 April (A) and 3 April (B). The dotted line represents the line assuming 5 absorbed photons are needed to produce a molecule of $\mathrm{O}_{2}$ by PSII

slopes of the lines give the conversion factor needed to convert ETR into rates of oxygen evolution, and are the reciprocal of the quantum requirement for charge separations in PSII. The values on 2 and 3 April are above the dashed line, which indicates the relationship when 5 absorbed photons are needed to produce a molecule of oxygen in PSII. Although the Z-scheme of photosynthesis requires 4 absorbed photons for PSII and 4 for
PSI, the minimum quantum requirement (QR) is likely to be closer to 10 (Mauzerall \& Greenbaum 1989). The values above the dashed line indicate a quantum requirement less than 5, and in all instances this seems to be the case, which is contradictory to our expectations. For the HRAP the lines at 7:00, 13:30 and 21:30 h on 2 April are identical, but the lines of the 2 nd half of the afternoon move upwards, indicating a decrease in

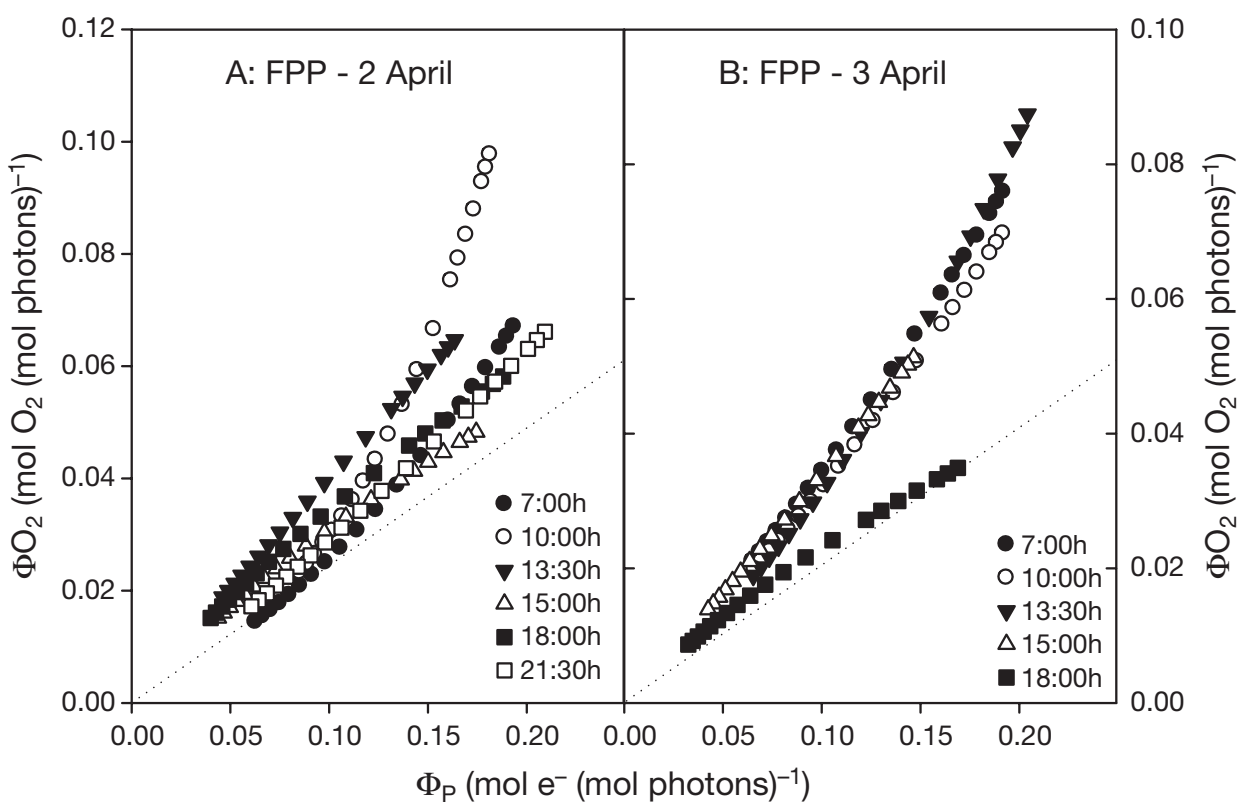

Fig. 9. Quantum efficiencies of oxygen evolution $\left(\Phi_{2}\right)$ plotted as a function of the quantum efficiencies of Photosystem II charge separation $\left(\Phi_{\mathrm{P}}\right)$ of the Nannochloropsis cells in the FPP on 2 April (A) and 3 April (B). The dotted line represents the line assuming 10 absorbed photons are needed to produce a molecule of $\mathrm{O}_{2}$ by PSII 
the quantum requirement. On 3 April the lines were closer together, indicating less variability in the quantum requirements during the day.

The QR of the cells in the FPP also showed QR below the minimum of 5 absorbed photons per molecule of $\mathrm{O}_{2}$ for PSII (Fig. 9), as all values were above the dashed line. Some non-linearity was also observed, especially in the 10:00 h sample on 2 April. This was caused by a large discrepancy between the $E_{\mathrm{k}}$ of ETR (425 $\mu \mathrm{mol}$ photons $\mathrm{m}^{-2} \mathrm{~s}^{-1}$ ) and the $E_{\mathrm{k}}$ of $\mathrm{O}_{2}$ evolution $(181 \mu \mathrm{mol}$ photons $\mathrm{m}^{-2} \mathrm{~s}^{-1}$ ). Like the situation observed for the HRAP the variability in the relationship showed more variation on 2 April than on 3 April, when all relationships showed good reproducibility as all lines were similar, with the exception of the 18:00 h sample. The relationship between $\Phi_{\mathrm{P}}$ and $\Phi \mathrm{O}_{2}$ approached 1:1 line indicating a quantum requirement of 5 absorbed photons per $\mathrm{O}_{2}$ molecule produced.

To further evaluate the slopes we plotted the reciprocal slopes (the QR) in Fig. 10. As mentioned above, all QR for PSII are below the minimum of 5, with the possible exception of the last FPP sample. In both the HRAP and FPP no clear pattern could be distinguished, although substantial variation in QR was observed. The increase in QR of the last measurement at 18:00 h in the FPP on 3 April is clearly visible. Despite the different optical conditions the QR of HRAP did not differ significantly (ANOVA, $\mathrm{p}=0.37$ ).

\section{DISCUSSION}

\section{Environmental (stress) conditions}

Due to the pond's larger volume compared with the FPP, the environmental changes were less in the HRAP pond where temperature fluctuations were smaller

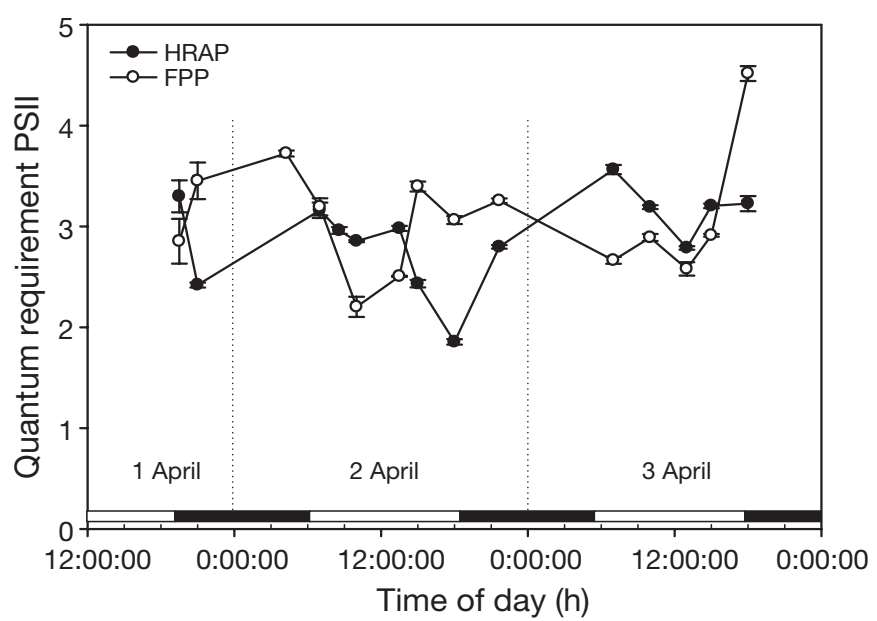

Fig. 10. Quantum requirements for oxygen evolution (reciprocal of slopes of Figs. 8 \& 9). The day/night period is given at the bottom of the panel as a light/dark bar than $15^{\circ} \mathrm{C}$ and $\mathrm{pH}$ changes did not exceed $2 \mathrm{pH}$ units, which is still considerable. However, temperature fluctuations in the FPP were much larger, with temperatures reaching higher than $40^{\circ} \mathrm{C}$ on 3 April due to the absence of wind and an insufficient cooling capacity inside the FPP. Without $\mathrm{pH}$ control the $\mathrm{pH}$ fluctuated nearly $3 \mathrm{pH}$ units, and the unusually high $\mathrm{pH}$ of 10 on Day 2 resulted in $\mathrm{CO}_{2}$ depletion as could be inferred from the photosynthesis parameters. Hence, despite the more constant optical conditions in the FPP, other environmental parameters were more variable.

\section{Biomass development and pigment content}

The average irradiance in the HRAP and FPP was estimated to be 93 and $222 \mu \mathrm{mol}$ photons $\mathrm{m}^{-2} \mathrm{~s}^{-1}$, respectively. As the cells in the FPP contained less chlorophyll than the cells used to estimate $k_{\mathrm{d}}$, the average irradiance might have been a little higher. However, because the FPP was situated vertically the average irradiance of $222 \mu \mathrm{mol}$ photons $\mathrm{m}^{-2} \mathrm{~s}^{-1}$ can be considered a maximum estimate as it will be influenced by the changing sun angle, which will increase path length. However, with so many cells the light field will become diffuse quickly, lessening the influence of a varying sun angle on the light path length. Despite the difference in average irradiance, the overall calculated growth rates for the HRAP culture was only a little lower $\left(0.13 \mathrm{~d}^{-1}\right)$ than growth rate in the FPP $\left(0.17 \mathrm{~d}^{-1}\right)$, and this difference was not significant.

Despite the similar pigment concentration, biomass (both as cell concentration or as DW) was approximately twice as high in the FPP than in the HRAP. Due to the higher irradiance the cells had a lower chl $a$ and carotenoid content, but a higher DW content per cell. Hence, despite uncertainties in the average irradiance, the HRAP showed shade acclimation and the FPP cells showed high light acclimation. The differences in cellular pigment content were reflected in a higher optical absorption cross section of the FPP cells, caused by a lower chl $a$ and carotenoid content per cell and a lesser degree of pigment packaging.

Because we did not perform HPLC analysis we cannot comment on the composition of the carotenoids, but the increase in the FPP in the carotenoid content as a result of the stressful temperature condition on Day 3 make it likely that this will be the result of an increase in protective carotenoids.

\section{Photosynthetic performance}

In general, the HRAP cells showed less variability in photosynthetic parameters (respiration and compensa- 
tion irradiance excepted, which showed an increase during the light period) than did the FPP cells, which showed clear signs of a decrease in $\mathrm{ETR}_{\max }$ and $P_{\max }$ during Day 2 when $\mathrm{CO}_{2}$ depletion occurred and during the high temperature stress conditions on Day 3. The HRAP culture also showed a shade-adapted acclimation pattern compared with the high light acclimation pattern of the FPP, as evidenced by higher ETR $_{\text {max }} E_{\mathrm{k}}$ and respiration values and by lower cellular chl a contents of the latter mass culture.

The cells in the FPP experienced severe stress conditions during Day 3, caused by the combination of a high irradiance (which was the same as the previous day) and a high temperature in the afternoon of Day 3. The results indicate that the decrease in photosynthetic activity is caused both by dynamic downregulation, most probably mediated by the xanthophyll cycle, and photodamage as 20 min dark adaptation was not long enough to relax $\mathrm{NPQ}$, leading to lowered $F_{\mathrm{v}} / F_{\mathrm{m}}$ values (Figs. $5 \& 6$ ). No recovery was observed in the $V_{j}$ and $V_{i}$ parameters, meaning that the $\mathrm{Q}_{\mathrm{B}}$ non-reducing PSII centres persisted. The role of NPQ could also be demonstrated by comparing the RLCs measured with and without dark adaptation. The decrease in $\mathrm{rETR}_{\max }$ was more pronounced in RLCs without dark adaptation and decreases in $\alpha_{\text {ETR }}$ were only visible in the RLCs without dark adaptation. Hence, the relaxation of NPQ during the dark adaptation period clearly affected the shape of the RLCs.

Despite the harsh conditions imposed by $\mathrm{CO}_{2}$ depletion on Day 2 and high temperature on Day 3, the primary processes of the light reaction remained closely coupled as we nearly always observed a linear relationship between $\Phi_{\mathrm{P}}$ and $\Phi_{\mathrm{O} 2}$. A high excitation pressure, which might be amplified by PSII damage, might induce an uncoupling between PSII charge separation and net oxygen evolution. This uncoupling might be caused by cyclic electron transport around PSII (Prasil et al. 1996, Lavaud 2007) or by the induction of alternative electron acceptors, especially the Mehler reaction, where donation of electrons by PSI via ferredoxin to $\mathrm{O}_{2}$ leads to the formation of hydrogen peroxide, which is subsequently converted back to water, leading to the water-water cycle (Asada 2000). This type of non-linearity has been frequently observed (e.g. see Flameling \& Kromkamp 1998) and seems to occur more in culture studies than in natural population studies (see Perkins et al. in press for a summary). Hence, in our study the Mehler reaction does not lead to net oxygen production, and we would see this as a decrease in $\Phi_{\mathrm{P}}$. This would cause a 'downward non-linearity' of the plots in Figs. 8 \& 9, and this clearly is not observed. This is also clear from the plots of the QR (Fig. 10), where the QR did not show a clear response during the light period in either the HRAP or FPP. The exception is 18:00 h sample from the FPP, which showed a clear rise in the QR. Hence, despite the stressful conditions, the light reactions of the photosynthetic pathway remain closely coupled and seem rather robust.

The calculated quantum requirements are below the theoretical minimum of 4 absorbed photons required by PSII to produce 1 molecule of $\mathrm{O}_{2}$. This obviously is not correct, and the easiest way to explain this is to assume that the antenna of PSII is larger than we assumed. Because we lack information on this we assumed that $50 \%$ of the light was absorbed by PSII (Gilbert et al. 2000), and this was corroborated by Suggett et al. (2004) who showed that for many algal taxa the fraction of light absorbed by PSII varied between 50 and $60 \%$, with the exception of cyanobacteria and the pelagophyte Aureococcus anophagefferens, although they weighted the absorption for the blue excitation light of the fast repetition rate fluorometer (FRRF). This is in contrast to the results obtained by Johnsen \& Sakshaug (2007) who showed that $F_{\text {II }}$ averaged 0.72. Assuming this value, the QR of the FPP would increase to $4.2 \pm 0.7$ and the QR of the HRAP to $4.4 \pm 0.8 \mathrm{~mol}$ absorbed photons per mol $\mathrm{O}_{2}$ produced. Thus, our computed QR requirements are too low most probably due to an underestimate of the true optical absorption cross section of PSII. In these calculations we ignored the possible effect of spectral differences between the red LED sources of the dual PAM and the halogen light source of the light pipette. For both light sources we calculated the fraction of the light that is absorbed (the so-called PUR/PAR ratio where PUR is photosynthetically usable radiation). Using a standard halogen light source this ratio was 0.26 and for the simulated red LED used by the PAM the PUR/PAR ratio was remarkably similar $(0.25)$. Hence, if we were to correct our estimates of the quantum efficiencies for the spectral differences of the light sources this would not affect our estimates of the quantum requirements as the same spectral correction factor needs to be applied.

As a result of the differences in photosynthetic performance and optical conditions experienced by the alga, the average biomass of FPP was about twice as high as that of the HRAP. The algae in the FPP also had a higher growth rate than those in the HRAP, although with the present data we could not prove that this higher growth rate was statistically significant. These differences were achieved with similar pigment concentrations in both culture systems. So, if biomass is the desired product the HRAP seems to be the method of choice as the larger depth will more than offset the lower concentration, and using the data from Table 1 the HRAP will yield $80 \mathrm{~g} \mathrm{~m}^{-2}$ whereas the FPP will yield $28 \mathrm{~g} \mathrm{~m}^{-2}$. As the systems were still growing these were minimum estimates. Also, the HRAP is less sensi- 
tive to mechanical failure. If high quality products are pursued, the FPP might be the method of choice. The FPP system is easier to manipulate with respect to products that are formed during special conditions. This can be exemplified in Fig. 3, where the temperature stress conditions induced formation of special carotenoids.

Acknowledgements. This study was carried out during the 8th International Workshop of the Group for Aquatic Primary Productivity (GAP) and the Batsheva de Rothschild Seminar on Gross and Net Primary Productivity held at the Interuniversity Institute for Marine Sciences, Eilat, Israel, in April 2008. We thank the Batsheva de Rothschild Foundation, Bar Ilan University, the Moshe Shilo Center for Marine Biogeochemistry and the staff of the Interuniversity Institute for funding and logistic support. Skillful help and technical assistance was provided by E. Ben-David, the National Mariculture Center (NCM), Israel Oceanographic and Limnological Research (IOLR). We thank M. Spiegel, M. Uhko and S. Bossibba for their generous support before and during this study. Partial funding (J.M. and J.K.) was also provided by projects MSM6007665808 and AV02 50200510 of the Ministry of Education Youth and Sports of the Czech Republic and by the project A608170601 of the Czech Academy of Sciences and project 521/09/0656 of the Czech Science Foundation. We thank 3 anonymous reviewers for their constructive remarks. This is NIOO publication 4586.

\section{LITERATURE CITED}

Asada K (2000) The water-water cycle as alternative photon and electron sinks. Philos Trans R Soc Lond B Biol Sci 355:1419-1431

Borowitzka M (1999) Commercial production of microalgae: ponds, tanks, tubes and fermenters. J Biotechnol 70: 313-321

> Chini Zittelli G, Lavista F, Bastianini A, Rodolfi L, Vincenzini M, Tredici MR (1999) Production of eicosapentaenoic acid by Nannochloropsis sp. cultures in outdoor tubular photobioreactors. J Biotechnol 70:299-312

> Dubinsky Z, Falkowski PG, Post AF, van Hes UM (1987) A system for measuring phytoplankton photosynthesis in a defined light field with an oxygen electrode. J Plankton Res 9:607-612

Flameling IA, Kromkamp J (1998) Light dependence of quantum yields for PSII charge separation and oxygen evolution in eucaryotic algae. Limnol Oceanogr 43:284-297

Gilbert M, Wilhelm C, Richter M (2000) Bio-optical modelling of oxygen evolution using in vivo fluorescence: comparison of measured and calculated photosynthesis/irradiance (P-I) curves in four representative phytoplankton species. J Plant Physiol 157:307-314

> Gonzalez C, Marciniak J, Villaverde S, Leon C, Garcia PA, Munoz R (2008) Efficient nutrient removal from swine manure in a tubular biofilm photo-bioreactor using algaebacteria consortia. Water Sci Technol 58:95-102

Gordon JM, Polle JEW (2007) Ultrahigh bioproductivity from algae. Appl Microbiol Biotechnol 76:969-975

Johnsen G, Sakshaug E (2007) Biooptical characteristics of PSII and PSI in 33 species (13 pigment groups) of marine phytoplankton, and the relevance for pulse-amplitudemodulated and fast-repetition-rate fluorometry. J Phycol 43:1236-1251
Klughammer C, Schreiber U (2008) Saturation pulse method for assessment of energy conversion in PSI. PAM Appl Notes 1:11-14

Kromkamp JC, Forster RM (2003) The use of variable fluorescence measurements in aquatic ecosystems: differences between multiple and single turnover measuring protocols and suggested terminology. Eur J Phycol 38: $103-112$

Lavaud J (2007) Fast regulation of photosynthesis in diatoms: mechanisms, evolution and ecophysiology. Funct Plant Sci Biotechnol 1:267-287

Lazár D (2006) The polyphasic chlorophyll a fluorescence rise measured under high intensity of exciting light. Funct Plant Biol 33:9-30

Lichtenthaler HK (1987) Chlorophylls and carotenoids, the pigments of photosynthetic biomembranes. In: Douce $\mathrm{R}$, Packer L (eds) Methods in enzymology, Vol 148. Academic Press, New York, p 350-382

Matthijs HCP, Balke H, VanHes UM, Kroon BMA, Mur LR, Binot RA (1996) Application of light-emitting diodes in bioreactors: flashing light effects and energy economy in algal culture (Chlorella pyrenoidosa). Biotechnol Bioeng 50:98-107

Mauzerall D, Greenbaum NL (1989) The absolute size of a photosynthetic unit. Biochim Biophys Acta 974:119-140

Neubauer C, Schreiber U (1987) The polyphasic rise of chlorophyll fluorescence upon onset of strong continuous illumination. I. Saturation characteristics and partial control by the Photosystem II acceptor side. Z Naturforsch Sect C J Biosci 42:1246-1254

> Nitsan Z, Mokady S, Sukenik A (1999) Enrichment of poultry products with $\Omega 3$ fatty acids by dietary supplementation with the alga Nannochloropsis and mantur oil. J Agric Food Chem 47:5127-5132

Perkins RG, Kromkamp JC, Serôdio J, Lavaud J and others (in press) The application of variable chlorophyll fluorescence to microphytobenthic biofilms. J Appl Phycol

Platt T, Jassby AD (1976) The relationship between photosynthesis and light for natural assemblages of coastal Maine phytoplankton. J Phycol 12:421-430

Prasil O, Kolber Z, Berry J, Falkowski PG (1996) Cyclic electron flow around photosystem II in vivo. Photosynth Res 48:395-410

> Richardson K, Beardall J, Raven JA (1983) Adaptation of unicellular algae to irradiance: an analysis of strategies. New Phytol 93:157-191

Shibata K, Benson AA, Calvin M (1954) The absorption spectra of suspensions of living micro-organisms. Biochim Biophys Acta 15:461-470

Strasser RJ, Srivastava A, Govindjee (1995) Polyphasic chlorophyll a fluorescence transient in plants and cyanobacteria. Photochem Photobiol 61:33-42

Strasser RJ, Tsimili-Michael M, Srivastava A (2004) Analysis of the chlorophyll a fluorescence transient. In: Papageorgiou GC, Govindjee (eds) Chlorophyll a fluorescence: a signature of photosynthesis. Advances in photosynthesis and respiration, Vol 19. Springer, Dordrecht, p 321-362

Suggett DJ, MacIntyre HL, Geider RJ (2004) Evaluation of biophysical and optical determinations of light absorption by photosystem II in phytoplankton. Limnol Oceanogr Methods 2:316-332

Sukenik A (1999). Eicosapentaenoic acid by the marine eustigmatophyte Nannochloropsis. In: Cohen Z (ed) Chemicals from microalgae. Taylor \& Francis, London, p 41-56

Sukenik A, Zmora O, Carmeli Y (1993) Biochemical quality of marine unicellular algae with special emphasis on lipid 
composition. II. Nannochloropsis sp. Aquaculture 117: 313-326

Sukenik A, Beardall J, Kromkamp JC, Kopecký J and others (2009) Photosynthetic performance of outdoor Nannochloropsis mass cultures under a wide range of environmental conditions. Aquat Microb Ecol 56:297-308

Takahashi S, Murata N (2008) How do environmental stresses accelerate photoinhibition? Trends Plant Sci 13:178-182

Webb WL, Newton M, Starr D (1974) Carbon dioxide exchange of Alnus rubra: a mathematical model. Oecologia 17:281-291

White AJ, Critchley C (1999) Rapid light curves: a new fluorescence method to assess the state of the photosynthetic apparatus. Photosynth Res 59:63-72

Editorial responsibility: Tom Berman,

Migdal, Israel
Zhang E, Wang B, Wang QH, Zhang SB, Zhao BD (2008) Ammonia-nitrogen and orthophosphate removal by immobilized Scenedesmus sp. isolated from municipal wastewater for potential use in tertiary treatment. Bioresour Technol 99:3787-3793

> Zhao B, Wanga J, Gong H, Wena X, Ren H, Lu C (2008) Effects of heat stress on PSII photochemistry in a cyanobacterium Spirulina platensis. Plant Sci 175: 556-564

Zhu YH, Jiang JG (2008) Continuous cultivation of Dunaliella salina in photobioreactor for the production of $\beta$-carotene. Eur Food Res Technol 227:953-959

Zou N, Richmond A (1999) Effect of light-path length in outdoor flat plate reactors on output rate of cell mass and of EPA in Nannochloropsis sp. J Biotechnol 70:351-356

Submitted: December 9, 2008; Accepted: May 4, 2009 Proofs received from author(s): July 14, 2009 